\title{
Epithelial-mesenchymal transition expression profiles as a prognostic factor for disease-free survival in hepatocellular carcinoma: Clinical significance of transforming growth factor- $\beta$ signaling
}

\author{
KOSUKE MIMA, HIROMITSU HAYASHI, HIDEYUKI KUROKI, SHIGEKI NAKAGAWA, HIROHISA OKABE, \\ AKIRA CHIKAMOTO, MASAYUKI WATANABE, TORU BEPPU and HIDEO BABA \\ Department of Gastroenterological Surgery, Graduate School of Medical Sciences, \\ Kumamoto University, Kumamoto 860-8556, Japan
}

Received July 5, 2012; Accepted October 3, 2012

DOI: $10.3892 / \mathrm{ol} .2012 .954$

\begin{abstract}
Epithelial-mesenchymal transition (EMT) plays a pivotal role in cancer invasion and metastasis, and transforming growth factor (TGF)- $\beta$ signaling is a potent inducer of EMT. However, the clinical significance of the correlation between EMT marker expression and TGF- $\beta$ signaling in hepatocellular carcinoma (HCC) patients remains unknown. In this study, immunohistochemistry was used to analyze the expression of EMT markers and phospho-Smad 2 nuclear positivity, and their association with clinicopathological

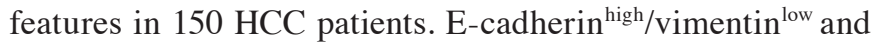
E-cadherin ${ }^{\text {low }} /$ vimentin $^{\text {high }}$ expression profiles were determined in $55(36.7 \%)$ and $21(14.0 \%)$ patients, respectively. The E-cadherin ${ }^{\text {low }} /$ vimentin ${ }^{\text {high }}$ expression profile was significantly correlated with poor tumor differentiation $(\mathrm{P}<0.001)$, vascular invasion $(\mathrm{P}=0.007)$ and extrahepatic recurrence following curative surgery $(\mathrm{P}=0.026)$. Furthermore, the E-cadherin ${ }^{\text {low }} /$ vimentin $^{\text {high }}$ expression profile was significantly correlated with shorter disease-free survival compared to E-cadherin ${ }^{\text {high} / v i m e n t i n ~}{ }^{\text {low }}(\mathrm{P}=0.002)$. Forty-one patients $(27.3 \%)$ were demonstrated to have high phospho-Smad2 nuclear positivity, which was significantly correlated with the E-cadherin ${ }^{\text {low }} /$ vimentin ${ }^{\text {high }}$ expression profile $(\mathrm{P}<0.001)$. In conclusion, this study suggests that EMT expression profiles are useful prognostic markers for disease-free survival in HCC patients, and that the E-cadherin ${ }^{\text {low }} /$ vimentin $^{\text {high }}$ expression profile is closely associated with high-grade malignant behavior such as tumoral vascular invasion and metastasis
\end{abstract}

Correspondence to: Professor Hideo Baba, Department of Gastroenterological Surgery, Graduate School of Medical Sciences, Kumamoto University, 1-1-1 Honjo, Kumamoto 860-8556, Japan E-mail: hdobaba@kumamoto-u.ac.jp

Key words: TGF- $\beta$, epithelial-mesenchymal transition, hepatocellular carcinoma, Smad2 in HCC. Additionally, TGF- $\beta$-mediated EMT may play an important role in the aggressiveness of $\mathrm{HCC}$.

\section{Introduction}

Hepatocellular carcinoma (HCC) is the fifth most prevalent and third most fatal type of cancer worldwide, and is currently diagnosed in over half a million people each year globally (1). Surgical resection is often performed as the most effective treatment for early-stage HCC. However, the 5-year risk of recurrence of $\mathrm{HCC}$ following resection is as high as $50-70 \%$, due to its high invasiveness and the frequent occurrence of intra- and /or extrahepatic metastases.

Epithelial-mesenchymal transition (EMT) has been shown to be a pivotal mechanism contributing to cancer invasion and metastasis, as epithelial cells lose their polarity and acquire the migratory properties of mesenchymal cells. The characteristic changes during EMT include the downregulation of epithelial markers, such as E-cadherin and the upregulation of mesenchymal markers such as vimentin (2). A correlation between the expression profiles of EMT and EMT inducers, and tumor recurrence and distant metastasis, has been demonstrated in certain types of cancer, including breast and colon cancer $(3,4)$. With regards to HCC, several EMT-related transcription factors such as Snail, Twist and Zinc finger E-box binding protein 1 (ZEB1) have been demonstrated to be involved in the process of EMT, and thus associated with a poor prognosis $(5,6)$.

Transforming growth factor (TGF)- $\beta$ signaling plays a central role in tumorigenesis and tumor progression by regulating many critical cellular processes, including cell proliferation, apoptosis and EMT. TGF- $\beta$ binds to type I and type II serine/threonine kinase receptors, resulting in the translocation of phosphorylated Smad proteins (phospho-Smad2 and 3) into the nucleus where they regulate the expression of various target genes (7). A previous study using HCC cell lines demonstrated that TGF- $\beta$ signaling triggered EMT, which was characterized by E-cadherin ${ }^{\text {low }} /$ vimentin $^{\text {high }}$ expression in vitro (8). However, the existence of a clinical association 
between the expression profiles of EMT markers and TGF- $\beta$ signaling, and its significance in HCC patients remains to be elucidated.

In the present study, we investigated the expression of the EMT markers E-cadherin and vimentin (epithelial and mesenchymal markers, respectively) and phospho-Smad 2 by immunohistochemical (IHC) analyses. The clinical significance of the expression profiles and TGF- $\beta$ signaling in HCC patients were further assessed.

\section{Materials and methods}

Patients and treatment. One-hundred and fifty primary HCC samples amongst 235 consecutive patients who underwent curative hepatic resection in the Department of Gastroenterological Surgery, Graduate School of Medical Sciences, Kumamoto University, between 2004 and 2007, were analyzed in this study. None of the patients had received any pre-operative anticancer treatment. The pathologic diagnoses and clinicopathological features were established based on the general guideline for primary liver cancer as defined by the Liver Cancer Study Group of Japan $(9,10)$ and the American Joint Committee on Cancer (AJCC)/International Union Against Cancer (UICC) staging system (11). The median follow-up duration following surgery was 44 months. Informed consent was obtained from all patients and the study was approved by the Human Ethics Review Committee of the Graduate School of Medicine, Kumamoto University (Kumamoto, Japan).

Immunohistochemistry and scoring. The sample processing and IHC procedures were performed as described in a previous study by Okabe et al (12). Endogenous peroxidase activity was blocked using 3\% hydrogen peroxide and the sections were incubated with diluted antibodies. A subsequent reaction was performed with a biotin-free horseradish peroxidase enzyme-labeled polymer from the Envision Plus detection system (Dako Japan Inc., Tokyo, Japan). Phospho-Smad2 antibody binding was detected using the Vectastain ABC Elite avidin/biotin/peroxidase kit (Vector Laboratories Inc., Burlingame, CA, USA). A positive reaction was visualized with the addition of diaminobenzidine solution, which was followed by counterstaining with Mayer's hematoxylin solution. Primary antibodies for E-cadherin (1:100 dilution; Japan BD, Tokyo, Japan), vimentin (1:50 dilution; Santa Cruz Biotechnology, Inc., Santa Cruz, CA, USA), and phospho-Smad2 (1:100 dilution; Cell Signaling Technology Japan, Tokyo, Japan) were used for this study. All IHC staining results were independently scored by two pathologists. The membranous E-cadherin and cytoplasmic vimentin expression was interpreted according to the guidelines published in a previous study by Yang et al (13). For membranous E-cadherin, cytoplasmic vimentin and phospho-Smad2-positive nuclei, the results were graded into categories from $0-3+$ as follows: 0 , no staining; $1+$, $1-25 \%$ staining; $2+, 26-50 \%$ staining and $3+,>50 \%$ of the specimen was stained. For membranous E-cadherin and phospho-Smad2-positive nuclei, the $2+$ and $3+$ samples were defined as positive IHC results. For cytoplasmic vimentin, the $3+$ specimens were defined as positive IHC results.

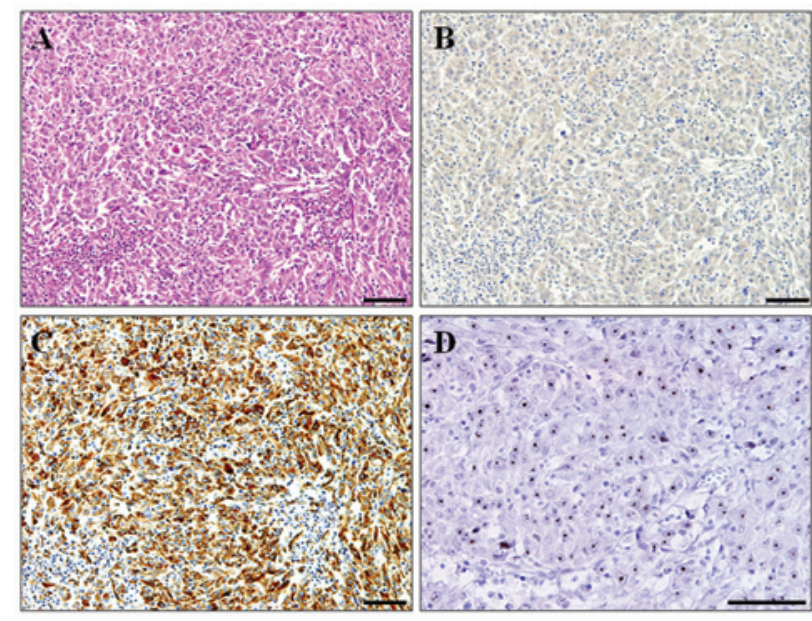

Figure 1. Immunohistochemical staining of E-cadherin, vimentin and phospho-Smad2 in hepatocellular carcinoma. (A) Representative case (hematoxylin-eosin staining) with (B) low E-cadherin, (C) high vimentin expression and (D) high phospho-Smad2 nuclear positivity. (Scale bars, $100 \mu \mathrm{m})$.

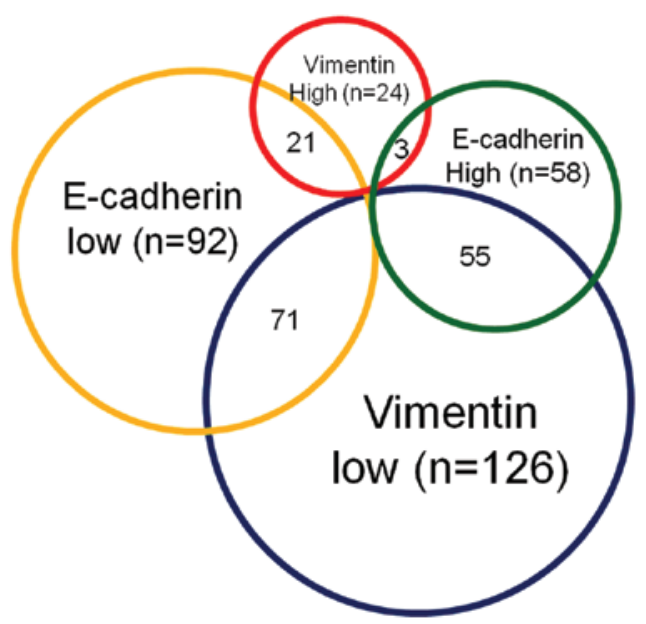

Figure 2. Venn diagram showing the number of hepatocellular carcinoma patients with E-cadherin/vimentin expression profiles using immunohistochemical analyses.

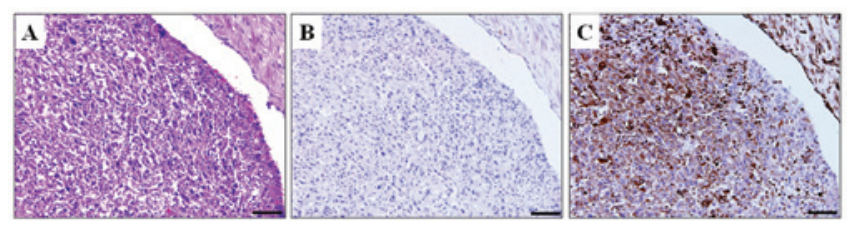

Figure 3. Immunohistochemical staining of E-cadherin and vimentin in portal vein tumor thrombosis in hepatocellular carcinoma . (A) Representative case (hematoxylin-eosin staining) with (B) low E-cadherin and (C) high vimentin expression. (Scale bars, $100 \mu \mathrm{m}$ ).

Statistical analyses. All experiments were performed in triplicate and the data shown are representative of the results. Categorical variables were compared using a $\chi^{2}$ test. Overall survival and disease-free survival were calculated using the Kaplan-Meier method and compared using a log-rank 

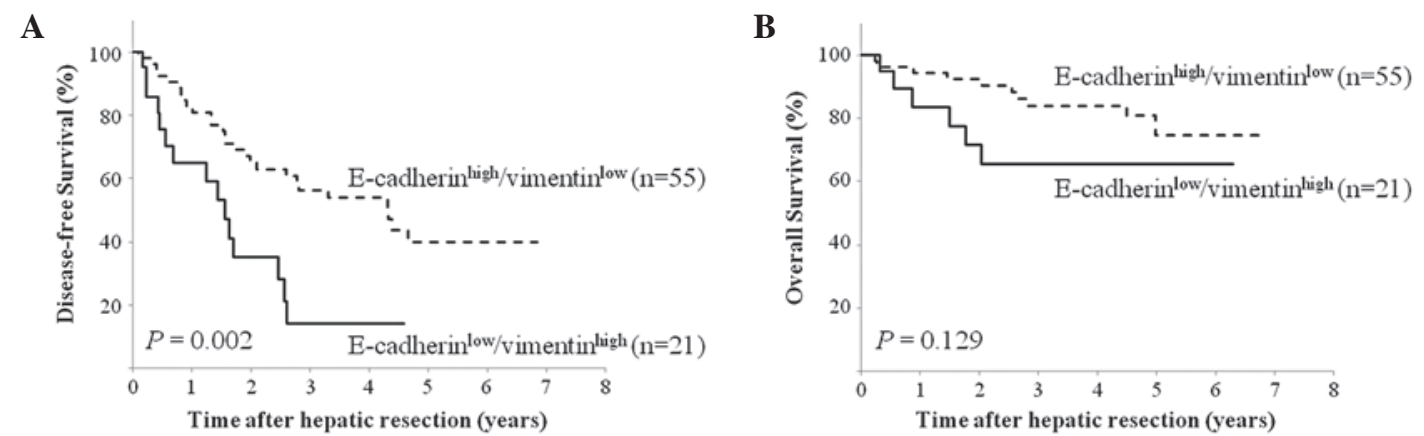

Figure 4. Disease-free and overall survival in hepatocellular carcinoma (HCC) patients according to E-cadherin and vimentin expression profiles. (A) Kaplan-Meier survival analyses of disease-free survival in HCC patients with E-cadherin ${ }^{\text {high }} /$ vimentin $^{\text {low }}$ and E-cadherin ${ }^{\text {low }} / v^{\text {vimentin }}{ }^{\text {high }}$ expression profiles, $^{\text {, }}$ compared using log-rank tests.(B) Kaplan-Meier survival analyses of overall survival in HCC patients with E-cadherin ${ }^{\text {high }} /$ vimentin $^{\text {low }}$ and E-cadherin ${ }^{\text {low }} / v$ imentin${ }^{\text {high }}$ expression profiles, compared using log-rank tests.

test. Statistical analyses were performed as indicated with a statistical analysis software program (Excel Statistics, Social Survey Research Information Co. Ltd., Tokyo, Japan). $\mathrm{P}<0.05$ was considered to indicate a statistically significant difference.

\section{Results}

EMT expression profile in HCC patients and its correlation with TGF- $\beta$ signaling. E-cadherin was mainly expressed in the tumor cell membrane, whereas vimentin was expressed in the tumor cytoplasm (Fig. 1). Among the 150 HCC patients, low and high E-cadherin expression levels were found in $92(61.3 \%)$ and $58(38.7 \%)$ patients, respectively. On the other hand, low and high vimentin expression levels were found in $126(84.0 \%)$ and $24(16.0 \%)$ patients, respectively. The EMT expression profiles in $150 \mathrm{HCC}$ patients are summarized in Fig. 2. In patients with HCC, a majority (47.3\%) exhibited an E-cadherin ${ }^{\text {low }} /$ vimentin $^{\text {low }}$ expression profile, whereas a minority $(2 \%)$ had an E-cadherin ${ }^{\text {high/vimentin }}{ }^{\text {high }}$ expression profile. Of the $150 \mathrm{HCC}$ patients, 55 (36.7\%) were revealed to have an E-cadherin ${ }^{\text {high} / v i m e n t i n ~}{ }^{\text {low }}$ expression profile (low ability of EMT), whereas 21 (14.0\%) were diagnosed with an E-cadherin ${ }^{\text {low }} /$ vimentin ${ }^{\text {high }}$ profile (high ability of EMT). TGF- $\beta$ signaling plays a central role in EMT; treatment of HCC cell lines with TGF- $\beta 1$ induced the E-cadherin ${ }^{\text {low }}$ vimentin $^{\text {high }}$ expression profile indicative of high ability of EMT (8). Therefore, we assessed the clinical relevance of TGF- $\beta$ signaling in patients with HCC by IHC analysis of phospho-Smad2; 41 (27.3\%) of 150 patients showed high phospho-Smad 2 nuclear positivity. To investigate the clinical association between the EMT marker profiles and TGF- $\beta$ signaling, phospho-Smad 2 nuclear positivity was compared between the subgroups with an E-cadherin ${ }^{\text {high} / v^{2}}$ imentin ${ }^{\text {low }}$ (low ability of EMT) or E-cadherin ${ }^{\text {low/vimentin }}{ }^{\text {high }}$ (high ability of EMT) expression profile. The E-cadherin ${ }^{\text {low }}$ vimentin $^{\text {high }}$ expression profile was significantly correlated with high phospho-Smad2 nuclear positivity, compared with the E-cadherin high/vimentin ${ }^{\text {low }}$ expression profile $(\mathrm{P}<0.001$; Table I). These findings suggest that TGF- $\beta$ signaling is closely associated with the EMT expression profile in HCC patients.
E-cadherin ${ }^{\text {low }} /$ vimentin $^{\text {high }}$ expression profile is associated with tumor invasion and metastasis in HCC patients. The correlation between the E-cadherin ${ }^{\text {low}} /$ vimentin ${ }^{\text {high }}$ expression profile and clinical invasiveness in HCC was further investigated by comparing the clinicopathological features between patients with E-cadherin ${ }^{\text {low }} /$ vimentin $^{\text {high }}$ (high ability of EMT) and E-cadherin $^{\text {high }} /$ vimentin $^{\text {low }}$ (low ability of EMT) expression profiles (Table I). The E-cadherin ${ }^{\text {low }} /$ vimentin $^{\text {high }}$ expression profile was significantly correlated with poor tumor differentiation $(\mathrm{P}<0.001)$, vascular invasion $(\mathrm{P}=0.007)$ and extrahepatic recurrence following curative surgery $(\mathrm{P}=0.026)$, compared with the E-cadherin ${ }^{\text {high} / v i m e n t i n ~}{ }^{\text {low }}$ expression profile. Cancer cells invading the portal vein, which is a distinctive characteristic of HCC, were negative for E-cadherin and positive for vimentin expression (Fig. 3). These findings suggest that EMT plays an important role in the invasive and metastatic phenotype of human HCC. In addition, we compared the clinicopathological features between patients with low and high phospho-Smad2 nuclear positivity. High phospho-Smad2 nuclear positivity was significantly correlated with the E-cadherin ${ }^{\text {low }} /$ vimentin ${ }^{\text {high }}$ expression profile $(\mathrm{P}<0.001)$. Although high phospho-Smad2 nuclear positivity was positively correlated with large tumor size $(\mathrm{P}=0.154)$, multiple tumors $(\mathrm{P}=0.110)$ and poor tumor differentiation $(\mathrm{P}=0.154)$ compared with low phospho-Smad2 nuclear positivity, the correlations were not statistically significant (Table II).

E-cadherin ${ }^{\text {low }} /$ vimentin ${ }^{\text {high }}$ expression profile is associated with poor disease-free survival in HCC patients. We investigated the prognostic implications of the E-cadherin ${ }^{\text {low/vimentin- }}$ high expression profile in HCC patients. The E-cadherin ${ }^{\text {low }}$ vimentin $^{\text {high }}$ expression profile was significantly correlated with shorter disease-free survival, compared to the E-cadherin ${ }^{\text {high/ }}$ vimentin $^{\text {low }}$ expression profile $(\mathrm{P}=0.002 ; \mathrm{Fig}$. $4 \mathrm{~A})$. E-cadherin ${ }^{\text {low }} /$ vimentin ${ }^{\text {high }}$ was also correlated with poorer overall survival than E-cadherin ${ }^{\text {high} / v}$ imentin ${ }^{\text {low; }}$, however, the difference was not statistically significant ( $\mathrm{P}=0.129$; Fig. 4B).

\section{Discussion}

This study demonstrated that a high ability of EMT (an E-cadherin ${ }^{\text {low }} /$ vimentin $^{\text {high }}$ expression profile) was closely 
Table I. Correlation between E-cadherin/vimentin expression and clinicopathological features in HCC patients.

$$
\text { E-cadherin }^{\text {high} / \text { vimentin }^{\text {low }}(\mathrm{n}=55) \quad \text { E-cadherin }}{ }^{\text {low }} / \text { vimentin }^{\text {high }}(\mathrm{n}=21)
$$

Age (years)

$\leq 60$
$>60$

20

35

Gender

Male

Female

HBs-Ag

Negative

Positive

$\mathrm{HCV}-\mathrm{Ab}$

Negative

Positive

Child-Pugh classification

A

B

AFP (ng/ml)

$\leq 20$

$>20$

PIVKA-II (mAU/ml)

$\leq 107$

$>107$

Tumor size $(\mathrm{cm})$

$\leq 3$

$>3$

Number of tumors

1

$\geq 2$

Tumor differentiation

Moderate/well

Poor

LCSGJ TNM stage

1 and 2

3 and 4

AJCC/UICC TNM stage

1 and 2

3 and 4

Vascular invasion $^{\mathrm{a}}$

Absent

Present

Extrahepatic recurrence ${ }^{b}$

Absent 53

Present

Phospho-Smad2 nuclear positivity

Negative

Positive
46

9

38

17

32

23

53

2

27

28

29

26

20

35

43

12

50

5

38

17

47

8

54

1

3

13

\section{8}

13

0.889

18

3

0.824

15

6

0.842

11

10

0.648

19

2

0.304

9

12

0.627

12

9

0.730

6

15

0.522

12

9

0.067

11

10

$<0.001$

10

11

0.083

16

5

17

4

0.007

17

4

0.026

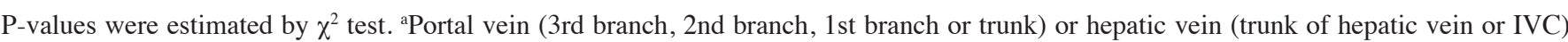

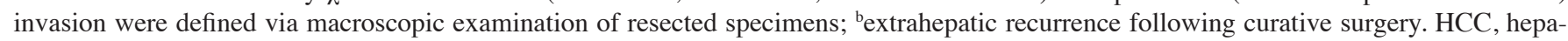
tocellular carcinoma; HBs-Ag, hepatitis B surface antigen; HCV-Ab, hepatitis C antibody; AFP, $\alpha$-fetoprotein; PIVKA II, protein-induced vitamin K absence-II; LCSGJ, Liver Cancer Study Group of Japan; AJCC/UICC, American Joint Committee on Cancer/International Union Against Cancer; phospho-Smad2, phosphorylated Smad2. Bold text denotes a statistically significant difference. 
Table II. Correlation between phospho-Smad2 nuclear positivity and clinicopathological features in HCC patients.

$$
\text { Low phospho-Smad2 }(\mathrm{n}=109) \quad \text { High phospho-Smad2 }(\mathrm{n}=41)
$$

P-value

Age (years)

$\leq 60$

$>60$

Gender

Male

Female

HBs-Ag

Negative

Positive

HCV-Ab

Negative

Positive

Child-Pugh classification

A

$\operatorname{AFP}(\mathrm{ng} / \mathrm{ml})$

$\leq 20$

$>20$

PIVKA-II (mAU/ml)

$$
\leq 107
$$

$>107$

Tumor size $(\mathrm{cm})$

$\leq 3$

$>3$

Number of tumors

$$
1
$$$$
\geq 2
$$

Tumor differentiation

Moderate/well

Poor

LCSGJ TNM stage

1 and 2

3 and 4

\section{AJCC/UICC TNM stage}

1 and 2

3 and 4

Vascular invasion ${ }^{\mathrm{a}}$

Absent

Present

Extrahepatic recurrence ${ }^{b}$
Absent
Present
E-cadherin ${ }^{\text {low }} /$ vimentin ${ }^{\text {high }}$
Negative
Positive

87

2

$$
36
$$

\section{0}

11

62

19

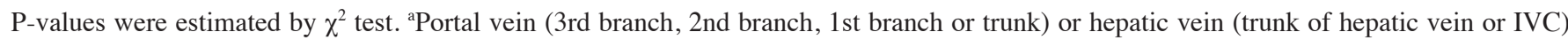
invasion were defined via macroscopic examination of resected specimens; ${ }^{b}$ extrahepatic recurrence following curative surgery. HCC, hepatocellular carcinoma; HBs-Ag, hepatitis B surface antigen; HCV-Ab, hepatitis C antibody; AFP, $\alpha$-fetoprotein; PIVKA II, protein-induced vitamin K absence-II; LCSGJ, Liver Cancer Study Group of Japan; AJCC/UICC, American Joint Committee on Cancer/International Union Against Cancer. Bold text denotes a statistically significant difference. 
correlated with high-grade malignant behavior, such as vascular invasion, extrahepatic recurrence and poor diseasefree survival, in HCC patients. In addition, the overexpression of vimentin, but not E-cadherin, was implicated in poorer disease-free survival in HCC patients. Vimentin expression has also been demonstrated to be correlated with the invasive phenotype in patients with gastric and breast cancer $(14,15)$. Cancer cell invasion of the portal vein is a clinically defined characteristic of HCC. Notably, the cancer cells invading the portal vein revealed a high ability of EMT, reflected in their E-cadherin ${ }^{\text {low }} /$ vimentin ${ }^{\text {high }}$ expression profile (Fig. 3). In contrast, analysis of another cohort of $123 \mathrm{HCC}$ samples revealed no correlation between either Snail/Twist overexpression or E-cadherin downregulation and vascular invasion (5). This study suggests that the loss of E-cadherin followed by the overexpression of vimentin may play a pivotal role in the invasive and metastatic phenotype and in the process of EMT, leading to unfavorable outcomes in patients with HCC.

TGF- $\beta$ signaling is known to be a potent EMT inducer and is strongly associated with cancer progression; EMT primes cancer cells for pulmonary metastasis and metastatic colonization in the bones $(16,17)$. In the present study, activated TGF- $\beta$ signaling indicated by phospho-Smad2 nuclear positivity was found in $27.3 \%$ (41/150) of HCC patients. Furthermore, phospho-Smad2 nuclear positivity was correlated with a high ability of EMT (E-cadherin ${ }^{\text {low/vimentin }}{ }^{\text {high }}$ ) in HCC patients. It has been demonstrated that TGF- $\beta 1$ is overexpressed in tumor cells, which is associated with a poor prognosis in patients with HCC $(18,19)$. These findings suggest that the activation of TGF- $\beta$ signaling is closely associated with EMT expression profiles in HCC patients. However, TGF- $\beta$ signaling was not activated in one-third of patients with an E-cadherin ${ }^{\text {low }}$ vimentin $^{\text {high }}$ expression profile. Although the precise mechanism of EMT induction in these patients remains unclear, it is possible that several transcription factors (such as Snail, Twist and ZEB1) as well as other molecules are also involved in the process of EMT and are therefore associated with a poor prognosis in $\mathrm{HCC}(5,6,20-27)$. Thus, several mechanisms, including TGF- $\beta$ signaling, may be involved in the process of EMT in HCC patients.

In conclusion, EMT expression profiles are useful prognostic markers for disease-free survival in HCC patients, and an E-cadherin ${ }^{\text {low }} /$ vimentin ${ }^{\text {high }}$ expression profile is closely associated with high-grade malignant behavior, such as tumoral vascular invasion and metastasis in HCC. TGF- $\beta$-mediated EMT may play a significant role in the aggressiveness of HCC.

\section{Acknowledgements}

The authors thank Keisuke Miyake and Naoko Yokoyama for their valuable technical assistance.

\section{References}

1. El-Serag HB: Hepatocellular carcinoma. N Engl J Med 365: 1118-1127, 2011.

2. Thiery JP, Acloque H, Huang RY and Nieto MA: Epithelialmesenchymal transitions in development and disease. Cell 139: 871-890, 2009

3. Moody SE, Perez D, Pan TC, et al: The transcriptional repressor Snail promotes mammary tumor recurrence. Cancer Cell 8 : 197-209, 2005
4. Shioiri M, Shida T, Koda K, et al: Slug expression is an independent prognostic parameter for poor survival in colorectal carcinoma patients. Br J Cancer 94: 1816-1822, 2006.

5. Yang MH, Chen CL, Chau GY, et al: Comprehensive analysis of the independent effect of twist and snail in promoting metastasis of hepatocellular carcinoma. Hepatology 50: 1464-1474, 2009.

6. Zhou YM, Cao L, Li B, et al: Clinicopathological significance of ZEB1 protein in patients with hepatocellular carcinoma. Ann Surg Oncol 19: 1700-1706, 2011.

7. Siegel PM and Massagué J: Cytostatic and apoptotic actions of TGF-beta in homeostasis and cancer. Nat Rev Cancer 3: 807-821, 2003.

8. Mima $\mathrm{K}$, Okabe $\mathrm{H}$, Ishimoto $\mathrm{T}$, et al: $\mathrm{CD} 44 \mathrm{~s}$ regulates the TGF- $\beta$-mediated mesenchymal phenotype and is associated with poor prognosis in patients with hepatocellular carcinoma. Cancer Res 72: 3414-3423, 2012.

9. Liver Cancer Study Group of Japan: The General Rules for the Clinical and Pathological Study of Primary Liver Cancer. Kanehara, Tokyo, 2009.

10. Minagawa M, Ikai I, Matsuyama Y, Yamaoka Y and Makuuchi M: Staging of hepatocellular carcinoma: assessment of the Japanese TNM and AJCC/UICC TNM systems in a cohort of 13,772 patients in Japan. Ann Surg 245: 909-922, 2007.

11. Vauthey JN, Lauwers GY, Esnaola NF, et al: Simplified staging for hepatocellular carcinoma. J Clin Oncol 20: 1527-1536, 2002.

12. Okabe H, Beppu T, Hayashi H, et al: Hepatic stellate cells accelerate the malignant behavior of cholangiocarcinoma cells. Ann Surg Oncol 18: 1175-1184, 2011.

13. Yang MH, Hsu DS, Wang HW, et al: Bmil is essential in Twist1-induced epithelial-mesenchymal transition. Nat Cell Biol 12: 982-992, 2010.

14. Iwatsuki M, Mimori K, Fukagawa $\mathrm{T}$, et al: The clinical significance of vimentin-expressing gastric cancer cells in bone marrow. Ann Surg Oncol 17: 2526-2533, 2010.

15. Vora HH, Patel NA, Rajvik KN, et al: Cytokeratin and vimentin expression in breast cancer. Int J Biol Markers 24: 38-46, 2009.

16. Padua D, Zhang XH, Wang Q, et al: TGFbeta primes breast tumors for lung metastasis seeding through angiopoietin-like 4. Cell 133: 66-77, 2008.

17. Yin JJ, Selander K, Chirgwin JM, et al: TGF-beta signaling blockade inhibits PTHrP secretion by breast cancer cells and bone metastases development. J Clin Invest 103: 197-206, 1999.

18. Bedossa P, Peltier E, Terris B, Franco D and Poynard T: Transforming growth factor-beta 1 (TGF-beta 1 ) and TGF-beta 1 receptors in normal, cirrhotic, and neoplastic human livers. Hepatology 21: 760-766, 1995.

19. Abou-Shady M, Baer HU, Friess H, et al: Transforming growth factor betas and their signaling receptors in human hepatocellular carcinoma. Am J Surg 177: 209-215, 1999.

20. Lee TK, Man K, Poon RT, et al: Signal transducers and activators of transcription $5 \mathrm{~b}$ activation enhances hepatocellular carcinoma aggressiveness through induction of epithelial-mesenchymal transition. Cancer Res 66: 9948-9956, 2006.

21. Chen L, Chan TH, Yuan YF, et al: CHD1L promotes hepatocellular carcinoma progression and metastasis in mice and is associated with these processes in human patients. J Clin Invest 120: 1178-1191, 2010.

22. Chung KY, Cheng IK, Ching AK, Chu JH, Lai PB and Wong N: Block of proliferation 1 (BOP1) plays an oncogenic role in hepatocellular carcinoma by promoting epithelial-to-mesenchymal transition. Hepatology 54: 307-318, 2011.

23. Fu J, Chen Y, Cao J, et al: p28GANK overexpression accelerates hepatocellular carcinoma invasiveness and metastasis via phosphoinositol 3-kinase/AKT/hypoxia-inducible factor-1alpha pathways. Hepatology 53: 181-192, 2011.

24. Kim H, Choi GH, Na DC, et al: Human hepatocellular carcinomas with "stemness"-related marker expression: keratin 19 expression and a poor prognosis. Hepatology 54: 1707-1717, 2011.

25. Wang J, Chen L, Li Y and Guan XY: Overexpression of cathepsin $\mathrm{Z}$ contributes to tumor metastasis by inducing epithelial-mesenchymal transition in hepatocellular carcinoma. PLoS One 6: e24967, 2011.

26. Yokomizo C, Yamaguchi K, Itoh Y, et al: High expression of p300 in HCC predicts shortened overall survival in association with enhanced epithelial mesenchymal transition of HCC cells. Cancer Lett 310: 140-147, 2011.

27. Zhu K, Dai Z, Pan Q, et al: Metadherin promotes hepatocellular carcinoma metastasis through induction of epithelial-mesenchymal transition. Clin Cancer Res 17: 7294-7302, 2011. 